\title{
$\overline{{ }^{n} \mathrm{D} A E}$ Optical imaging techniques for rice diseases detection: A review
}

Nur Azizah Bachika, Norhashila Hashima,b, Aimrun Wayayoka, Hasfalina Che Mana, Maimunah Mohd Alia,

${ }^{a}$ Department of Biological and Agricultural Engineering, Faculty of Engineering, Universiti Putra Malaysia, 43400 UPM Serdang, Selangor, Malaysia

${ }^{b}$ SMART Farming Technology Research Centre (SFTRC), Faculty of Engineering, Universiti Putra Malaysia, 43400 Serdang, Selangor, Malaysia.

\section{ARTICLE HISTORY Abstract}

Received: 2 March 2020

Received in revised form: 9 March

2020

Accepted: 10 March 2020

Available Online: 12 March 2020

\section{Keywords}

Computer vision

Rice diseases

Image processing

Hyperspectral imaging

Near-infrared spectroscopy

Remote sensing
Rice diseases have caused great economic losses to farmers in rice cultivation. The current assessment of rice disease evaluation still relies on manual, subjective, and laborious techniques. The manual and subjective evaluations lead to uncertainties since some diseases have almost similar characterisation. The applications of immunological, molecular, and microscope techniques are time-consuming, costly, and skills dependent. Thus, optical techniques are recommended to facilitate the control of diseases through their feasibility, rapidity, and accuracy, which can lead to better management strategies, besides improving production activity. These techniques for detecting and monitoring the diseases are important for precaution and prevention action. The present review discusses the existing and potential optical techniques for the detection of rice diseases. The techniques include optical imaging that consists of computer vision, spectroscopy, multispectral imaging, hyperspectral imaging (HSI), and remote sensing. Thus, this work presents in-depth information related to the nondestructive and potential applications of optical imaging techniques for rice disease detection.

\section{Introduction}

Rice is one of the most vital cereal plants and staple food in the world (Kurniawati et al., 2009). However, infection of diseases caused by pathogens, which include bacteria, fungi, and viruses, may cause great economic losses to farmers as rice plant is easily susceptible to diseases and infections (Yao et al., 2009). There are many types of rice diseases at present, but the most popular ones are rice blast and brown spot. Rice plant diseases, such as bacterial leaf streak, blast, brown spot, leaf scald, narrow brown leaf spot, red stripe, sheath blight, and tungro, were classified by the Rice Knowledge Bank as the diseases that impose on the leaves (Surendrababu et al., 2014). Every rice plant disease has its own unique pattern, but some of them, such as blast, brown spot, tungro, leaf scald, and red stripe are very similar in which they mimic the pattern of natural ageing of leaves. For this reason, recognition of diseases at an early stage is vital for early prevention and precautions.

Recently, the detection of diseases by farmers mainly relies on their experiences, guidance from books, and help from the experts for identification. However, these manual methods are only suitable for detecting typical diseases. In some cases, diseases share similar spots, but different spots can also reflect the same diseases due to different rice varieties (Ali et al., 2019). This complexity of identifying rice diseases can lead to incorrect measurement and use of wrong pesticides. Besides, the detection of growth of diseases in the plant at an early stage is important so that farmers can take necessary actions for preventing the diseases from spreading to the other parts of the field (Suman \& Dhruvakumar, 2015).

Some methods of detection of rice diseases that have been used to date are immunological and molecular methods, such as polymerase chain reaction (PCR), enzyme-linked immunosorbent assay (ELISA), and microscopy (Sharma \& Dasgupta, 2012). These methods are reliably accurate, but time consuming, costly, and need professional expertise. Consequently, they are impossible to be applied in real-time condition. In addition, technology like PCR has several limitations, which are complicated and provides uncertainty *Corresponding author email: maimunah_mohdali@ymail.com results, such as the uneven distribution of pathogens in host plants, presence of PCR inhibitors in the extracted DNA/RNA, and low pathogen concentration (Pereira et al., 2010). Since the diseases need to be identified quickly to reduce the impact of the pathogen attack and to control the diseases, optical imaging techniques, such as computer vision, spectroscopy, hyperspectral imaging (HSI), and remote sensing have displayed the great potential to be applied in rice industries. Hence, this paper provides information on the application of optical imaging techniques in the detection of rice diseases. The paper also discusses the fundamental theory that depicts the development of optical imaging techniques.

\section{Optical properties of plant tissues}

The most investigated plant part for the optical properties of plant tissues is the leaf due to its important role in photosynthesis. Besides, the interaction of electromagnetic radiations on the plant leaves, which are transmission, absorption, and reflection, strongly depends on the physical and the chemical characteristics of leaves (Steiner et al., 2012). Optical properties of leaf tissue consist of absorption, scattering, transmission and reflection (Mahesh et al., 2015). Absorption is the process when the energy of a photon is taken up by matter while the transmission is the process of light passing through the matter. On the other hands, the reflectance is the process when incident illumination reacts with matter and returns from its surface which converted to radiant energy. As a result of leaf reflectance of sunlight, multiple interactions occurred on the leaf such as interaction with cell structure and pigments like chlorophyll (Kanda et al., 2007). In contrast, light scattering is the process when the light in the form of propagating energy is scattered as the deflection of a ray from a straight path. During light propagation in the tissue, the light carries useful information on the cellular characteristics and structures (Hashim et al., 2013). As absorption of radiant energy is induced by water content and chemistry of the leaf, light scattering is induced by the leaf surface and internal cellular. 
The optical domains which range from $400 \mathrm{~nm}$ to $2500 \mathrm{~nm}$ are divided into three parts which are visible $(400 \mathrm{~nm}-800 \mathrm{~nm})$, near-infrared $(800-1100 \mathrm{~nm})$ and short wave infrared (1100$2500 \mathrm{~nm}$ ) (Valente et al., 2009). Visible range is characterized by strong absorption of light by photosynthesis pigments in the green leaf (Buschmann et al., 2012). In near-infrared range (800-1100nm), absorption is limited to dry matter but multiple scattering within the leaf due to a fraction of air spaces and internal structure drives the reflectance and transmittance levels. Middle infrared range (1100-2500nm) has strong absorption influenced by water in a fresh leaf and dry matter when the leaf wilts. Level of light absorption is influenced by the amount of chlorophyll in the parenchyma and spongy mesophyll. Low reflectance in the visible range is due to absorption by photoactive plant pigments. Leaf pigments i.e. chlorophyll a and chlorophyll b absorbs blue light (400nm$495 \mathrm{~nm})$ and red light $(620 \mathrm{~nm}-700 \mathrm{~nm})$ while carotenoids absorb only blue light (400nm-495nm) (Yang, 2010). These pigments transfer the absorbed energy into the photosynthetic electron chain. On the other hand, in the near-infrared range, leaf internal structure, leaf anatomy and epidermal surface characteristics influence the reflectance of light.

Multiple internal scattering processes within the leaf tissue and direct reflection on the leaf surface causing high reflection in the near-infrared region. Correspondingly, minor absorption in the near-infrared region is influenced by leaf biochemical compounds like cellulose, carbohydrates and lignin (Singh et al., 2012). Moreover, multiple interactions those take place between incoming irradiation and biophysical, such as tissue structure and leaf surface, as well as biochemical characteristics, such as water and pigments, are the results of reflectance on the leaves. However, pathogens activities also may cause physiological changes in plant metabolism that result in the appearance of disease symptoms. Appearances due to modifications in pigments, tissues, and water content of plants can be related to the changes in optical properties.

\section{Optical imaging techniques for rice diseases detection}

\subsection{Computer vision}

Computer vision has been used for many applications in the agricultural sector. These applications include the detection of disease or insect-damage, the detection of nutrient deficiencies, the estimation of quality in agricultural products, as well as in the grading and sorting processes for fruits and vegetables (Brosnan \& Sun, 2004; Chen et al., 2012; Hu et al., 2010; Narendra \& Hareesh, 2010; Patel et al., 2012). In paddy, the system has been used to distinguish head rice from broken rice based on the rice shape, besides evaluating the quality of rice (Maheshwari, 2013; Renugambal \& Senthilraja, 2015). It is a cost-effective system, non-destructive, rapid, accurate, and automatic, which can be applied in the laboratory or at the field as an automated system (Mohd Ali et al., 2020). Moreover, computer vision system processes and analyses digital images in determining specific attributes, such as shape or colours that yield to object recognition via automatic processing of a digital image. Besides, the computer vision system electronically perceives and evaluates an image by emulating the function of human vision.

The basic components of the computer vision system mostly consist of an imaging sensor, the light source for illumination, computer or chip as a processing device for image processing algorithms, inspecting flat or holder to hold the plant sample, and a liquid-crystal display (LCD) screen for data output. The main components of the computer vision system are illustrated in Figure 1. The imaging sensors can range from lowcost high-resolution digital cameras to expensive chargedcouple device (CCD) cameras (Casanova et al., 2014). However, it has been recommended to use a CCD camera as the imaging sensor as it produces high-quality images, which later could reduce image pre-processing works. Good illumination also helps to reduce noise in the image that simplifies the segmentation process. In addition, when designing an illumination system, some aspects, such as lamp type, location, and colour quality, must be considered. When surface feature extraction is needed, front lighting is recommended to be used, whereas when sub-surface feature analysis or critical edge dimensioning is needed, backlighting is recommended (Brosnan \& Sun, 2004). Additionally, the selection of light sources is one of the important steps as it affects the quality and the image analysis performance. Light sources that can be used include incandescent, lasers, fluorescent, infrared lamps, and X-ray tubes. Thus, the computer vision system component may be added or modified based on the user function.

During the image acquisition process, the camera captures the image and sends the acquired data to the computer. The computer would keep the image, which will be further used for image analysis. Image acquisition is the process of obtaining the images. The acquisition of images is commonly done with a white or black background in order to avoid reflections while capturing the images. During the acquisition process, the angle of the camera, the intensity of light, the distance between camera and sample, as well as the sample position or point on the sample, should be consistent in order to obtain consistent and accurate images for analysis (Tan et al., 2014). For instance, Kurniawati et al. (2009a) applied Otsu, variable, and global

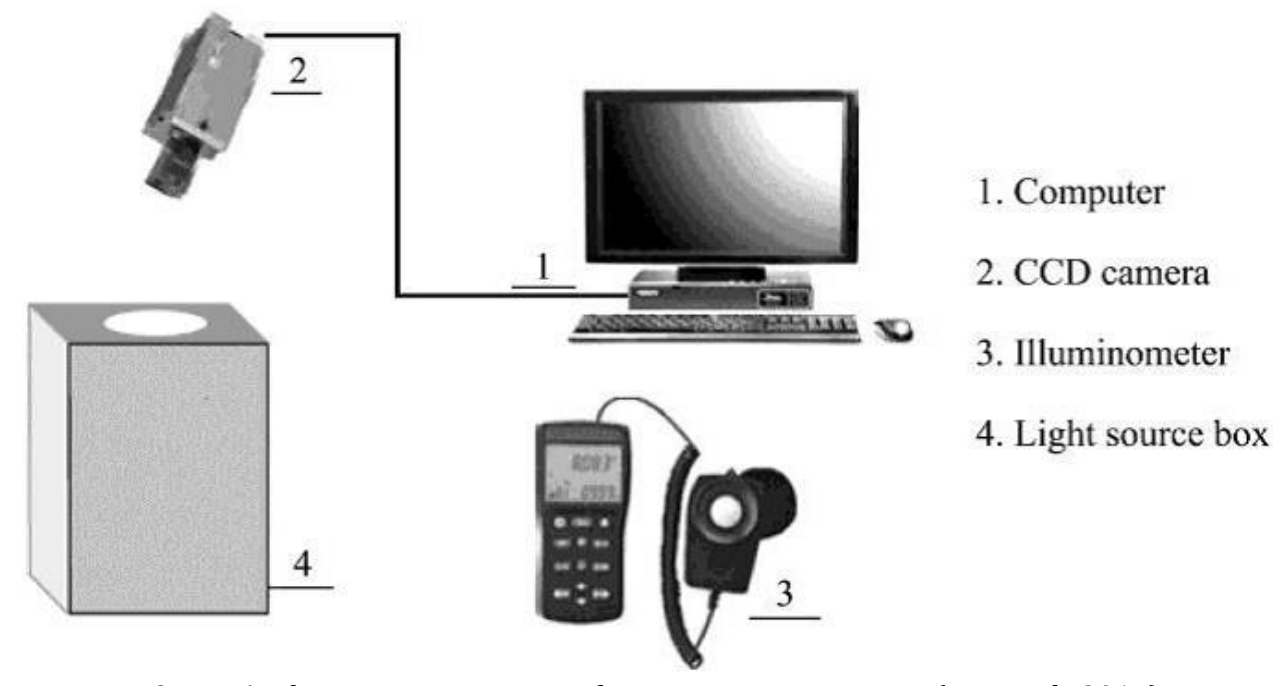

Figure 1. The main components of computer vision system (Tan et al., 2014) 
thresholding methods for diagnosing blast, brown spot, and narrow brown spot diseases. The results indicated that the Otsu method did not perform well with only $44 \%$ of segmentation accuracy rate. Besides, during the segmentation process, the determination of threshold value is important to obtain both accurate segmentation and high percentage of classification.

On the other hand, colour-based segmentation segments colours in an image. An example of the colour-based segmentation method is the K-means clustering. K-means clustering is also a classification method that separates data into k-mutually exclusive clusters and returns the index of the cluster to which it has assigned to each observation (Tan et al., 2014). K-means clustering operates on actual observations and creates a single level of clusters. Phadikar et al. (2013) applied the K-means clustering method to separate accurately between non-infected and infected regions of the images before using the rough set theory (RST) to minimize loss of information and rule base classifier to distinguish brown spot, blast, sheath rot, and bacterial blight diseases. The finding revealed more than $90 \%$ averages of accuracies were obtained among the classifiers.

Asfarian et al. (2013) identified the four major rice diseases, which are rice leaf blast, brown spot, bacterial leaf blight, and tungro disease by using fractal descriptors based on Fourier spectrum to analyse the texture of the diseased lesions. The classification results showed that the overall accuracies had been $91.80 \%, 92.31 \%, 96.25 \%, 83.00 \%$, and $97.96 \%$ for brown spots, bacterial leaf blight, leaf blast, and tungro disease, respectively. Phadikar et al. (2013) classified rice leaf brown spot, rice blast, and sheath rot, whereas bacterial blight diseases by using feature selection and rule generation techniques (Figure 2). The rule base classifier was built to classify the diseases by using the extracted features from the infected region of images and the results revealed more than $90 \%$ of chances with different average accuracies among other classifiers. Otsu method was used for automatic segmentation of rice planthopper by adding the gray-level information based on the segmented images (Hongwei et al., 2016). Chung et al. (2016) studied the application of computer vision to classify between healthy and infected rice seedlings with Bakanae disease. The SVM classifier was used to distinguish between the healthy and infected rice seedlings with an accuracy rate of $88 \%$.

Ghyar and Birajdar (2017) investigated disease detection in rice infected by pests using computer vision system. Support vector machine (SVM) and artificial neural network (ANN) classifiers obtained classification accuracies of $93 \%$ and $88 \%$, respectively. In previous work, Tanmayee (2017) developed a rice crop monitoring system via computer vision to eliminate the spread of disease infection. It was indicated that the system was able to reduce the pesticide wastage in the rice field by tracking the periodic images based on the crop area. Abu Bakar et al. (2018) investigated the early detection of rice leaf blast using image processing approach in order to distinguish between three severity levels (infection, spreading, and worst). Similarly, Suman \& Dhruvakumar (2015) used SVM classifier for the classification of brown spot, rice blast, and bacterial leaf blight in rice using histogram plot from the segmented images using computer vision system. The summary of rice diseases detection by using image processing techniques is shown in Table 1.

\subsection{Multispectral imaging}

Multispectral imaging is another technique that combines the spectroscopic approach and conventional imaging from the selected samples in order to obtain information in terms of spectral and spatial datasets. Multispectral imaging requires no sample preparation, rapid, non-destructive, and suitable for assessing food safety and quality evaluation of agricultural products (Liu et al., 2015; Lu, 2004; Shrestha \& Hardeberg, 2014). Besides, this technology is able to determine multiple components simultaneously for measuring quality assurance. Zhang et al. (2018) used a quadrotor unmanned aerial vehicle (UAV) combined with the multispectral camera to obtain

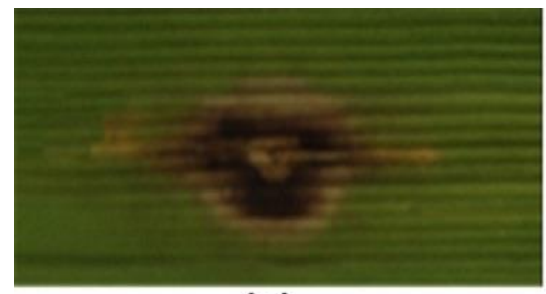

(a)

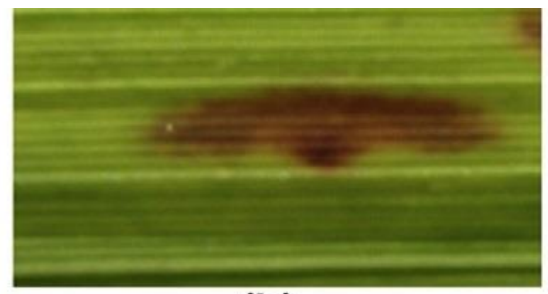

(b)

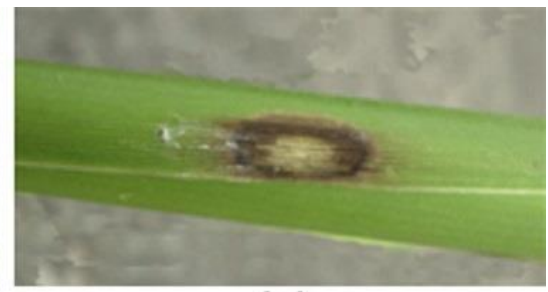

(c)

Figure 2. Infected rice leaves (a) rice blast, (b) brown spot and (c) sheath rot (Phadikar et al., 2013)

Table 1. Studies for rice diseases detection by using computer vision system

\begin{tabular}{|c|c|c|c|}
\hline Equipment & Types of paddy diseases & Results and accuracies & References \\
\hline $\begin{array}{l}\text { Automatic computer vision-based } \\
\text { system }\end{array}$ & $\begin{array}{l}\text { Bacterial leaf blight, rice } \\
\text { sheath blight, rice blast }\end{array}$ & SVM-92.5 \% & $\begin{array}{l}\text { Ghyar and Birajdar } \\
\text { (2017) }\end{array}$ \\
\hline $\begin{array}{l}\text { Web camera USB } 2.0 \text { with image } \\
\text { sensor }\end{array}$ & Bacterial blight & $\begin{array}{l}\text { Reduces the need for pesticides to the } \\
\text { entire farm }\end{array}$ & Tanmayee (2017) \\
\hline Flatbed colour scanners & Bakanae disease & SVM-87.9 \% & Chung et al. (2016) \\
\hline $\begin{array}{l}\text { Photo-axis feature of yellow and } \\
\text { green with fluorescent mercury } \\
\text { lamps }\end{array}$ & Rice planthoppers & $\begin{array}{l}\text { Iteration time compared to traditional } \\
\text { method is reduced by half }(312 \mathrm{~ms})\end{array}$ & Hongwei et al. (2016) \\
\hline Multi-level colour image camera & Rice leaf blast & $\begin{array}{l}\text { Able to detect the disease based in } \\
\text { uncontrol environment }\end{array}$ & $\begin{array}{l}\text { Abu Bakar et al. } \\
\text { (2018) }\end{array}$ \\
\hline $\begin{array}{l}\text { Nikon COOLPIX P4 digital camera, } \\
\text { Canon Power Shot G2 digital } \\
\text { camera }\end{array}$ & $\begin{array}{l}\text { Bacterial leaf blight, brown } \\
\text { spot, narrow } \\
\text { brown spot, rice blast }\end{array}$ & SVM-70 \% & $\begin{array}{l}\text { Suman and } \\
\text { Dhruvakumar (2015) }\end{array}$ \\
\hline Camera digital & $\begin{array}{l}\text { Rice blast, brown spot, } \\
\text { bacterial leaf blight, tungro }\end{array}$ & PNN- $91.80 \%$ & Asfarian et al. (2013) \\
\hline Camera digital & $\begin{array}{l}\text { Brown spot, rice blast, } \\
\text { sheath rot, bacterial blight }\end{array}$ & Rule generation algorithm- $90 \%$ & Phadikar et al. (2013) \\
\hline
\end{tabular}

SVM, support vector machines; PNN, propagation neural network. 
imaginary data to detect rice sheath blight disease. The multispectral images were analysed by using colour space transformation that can measure the infected disease in the field. The findings indicated that the different disease levels can be classified based on the multispectral images with a classification accuracy of $63 \%$. The utilisation of UAV coupled with multispectral imaging proved that it can be applied in a bigger scale plot in the detection of rice sheath blight disease.

Kobayashi et al. (2007) reported the potential of the airborne multispectral scanner in the detection of panicle blast in rice. The rice reflectance was selected at the wavebands of 485 and $675 \mathrm{~nm}$ in the visible region. In this study, the indicator of the panicle blast was determined via the rice reflectance ratios. At the yellow-ripe stage, the detection of panicle blast was effective in the near-infrared reflectance. Meanwhile, the wavebands of 530-570 $\mathrm{nm}$ and $650-700 \mathrm{~nm}$ were selected as the best region for panicle blast detection at the dough stage. In another research, Qin and Zhang (2005) discussed the application of multispectral remote sensing in the detection of rice sheath blight in the field. Based on the imaginary data, the identification accuracy increased when the infection obtained disease index higher than 35 . It was concluded that the multispectral images with greater waveband resolution were chosen to discriminate between the healthy and infected plants. On the other hand, Liu et al. (2014) applied multispectral imaging to determine transgenic rice seeds from the nontransgenic seeds in order to compare classification performance using chemometric analysis. The results indicated that the least squares-support vector machines (LS-SVM) could classify between the transgenic and non-transgenic rice seeds with a classification accuracy of up to $100 \%$. Hence, the multispectral imaging coupled with chemometric analysis delivers a promising approach to provide classification performance with high efficiency for future research.

\subsection{Hyperspectral Imaging}

Hyperspectral imaging (HSI) is an extension of multispectral imaging, which can acquire both spectral and spatial information from samples. It is a combination of spectroscopy and image processing (El Masry et al., 2011). HSI provides a large dataset called data cube that can provide a complete and reliable analysis of external characteristics and intrinsic properties of samples (Adebayo et al., 2016). Besides, HSI provides an abundance of spectral information where necessary features can be extracted for further analysis by using image processing procedure, but precaution should be taken to avoid loss of important information in the original hyperspectral data. The hyperspectral images of samples can produce reflectance, absorbance or both spectra, which can be used for chemometric analyses. In addition, NIR radiation that penetrates well into samples can generate diffuse reflectance spectra. Moreover, when there is no reflection in the absorbed radiation of samples, absorbance spectra are produced. Thus, sample characterization and concentration determination can be generated from absorbance spectra.

Basically, hyperspectral data are called hypercube which arranged as a three-dimensional (3-D) cube of two spatial dimensions and one spectral dimension (Aleixos et al., 2002). Each hyperspectral image cube consists of 50-300 images that are acquired at different wavelengths, with $1-10 \mathrm{~nm}$ of spectral resolution from a specific wavelength region (e.g. 750-2500nm). In addition, hyperspectral data can be transformed into radiometric quantities, such as transmittance, absorbance, and reflectance, which then can be related to the chemical composition or the physical characteristics of samples. The actual configuration of the HSI system depends on the kind of approach used. Overall, the basic parts of the HSI system consist of a charge-coupled device (CCD) camera, a detector, a frame grabber, a filter, an illumination system (halogen lights), and a computer. The detection of a limited number of photons entering the detector requires a highly sensitive CCD camera with a high signal-to-noise ratio, while to require hyperspectral images effectively, the power illumination system is needed. In addition, large capacity hard disk and memory are required for processing hyperspectral information with a high-speed computer.

Both visible and NIR HSI have been widely used for monitoring the quality of food and agricultural products. They have been used in the detection of infected plants to classify agricultural materials by determining moisture content, total soluble solids content, and acidity in strawberries (El Masry et al., 2007) to classify maize kernel hardness (Williams et al.,2009), to detect early bruises in apples (Baranowski et al., 2012), to earlier detect infected wheat (Bauriegel \& Herppich, 2014), to discriminate maturity level and colour evolution in apples under different storage conditions (Garrido-Novell et al., 2012), to detect decay in citrus fruit (Lorente et al., 2013) and to detect citrus cancer (Qin et al., 2009). This system uses advanced procedures for image processing and analysis like other techniques, which include PCA, partial least squares (PLS), neural networks, linear discriminant analysis, and SVM.

Yang et al. (2012) used the NIR ( 900 to $1700 \mathrm{~nm}$ ) HSI system for early detection of rice blast disease at the seedling stage in the Nipponbare rice variety. The HSI, which is known as hypercube, was collected in the diffused reflectance mode that consisted of two types of hypercubes, i.e. healthy and infected rice seedlings. PCA was used for data reduction, while stepwise discriminant analysis was employed to select variables with a significant contribution for the desired classification, and linear discriminant analysis (LDA) classifier models were carried out based on the selected variables. Furthermore, five significant wavelengths $(1188,1339,1377,1432$, and 1614nm) were selected, whereas PCA, LDA, and stepwise discriminant analysis scores of the images were recalculated by using the five selected wavelengths. The PCA score measurement from the selected wavelengths and full-spectrum had been used to develop and compare classification models. Based on the LDA classifier, features extracted from full spectrum (950 to $1650 \mathrm{~nm}$ ) images displayed an overall accuracy of $92 \%$ in the validation set, while features extracted from five wavebands exhibited an overall accuracy of $80 \%$ in the validation set. From this study, a few specific wavebands showed the potential for detection of rice blast and may be possible to build a low-cost imaging system to identify the rice blast disease based on canopy information imagery.

Meanwhile, prediction and grading of rice panicle blast level based on the concept of the "bag of textons" (BoSW) model for HSI data representation were carried by Huang et al. (2015). This study employed the BoSW model to analyse the 3D HSI of rice panicles from more than 50 cultivars collected in two different seasons from the same field under the natural condition to enable blast disease grading. A hyperspectral imaging system was used to acquire 312 rice panicles images that had been naturally infected with six different blast infection levels; $0,1,3,5,7$, and 9 , which were collected in two batches at the yellow-ripe stage. This study proposed the concept of a spectrum prototype and the statistical distribution of the spectrum prototype was used to grade the rice blast severity level with 186 samples for training and 126 for testing for the combination of the two batches. The results showed that the proposed method graded rice panicle blast with a classification accuracy of $81.41 \%$ for six-class grading, while $96.40 \%$ for twoclass grading in the validation datasets. All the baseline methods using SVM to construct the classifying model and the results showed that BoSW method offered the best performance. 


\subsection{Spectroscopy-based techniques}

Spectroscopy is a study of the interaction between radiation and matter as a function of wavelength. The spectroscopic technique that is used to assess the concentration or the amount of a sample is known as spectrometry, while a spectrometer or a spectrograph is the instrument that performs the measurement (Lorente et al., 2015). Similar to computer vision, spectroscopy, which includes ultraviolet (UV), visible (VIS), and near-infrared (NIR) range, offers many advantages, such as rapidity and simplicity in data collection, can be easily automated since spectroscopy is a system that requires minimal sample processing and preparation before analysis, involves probe to acquire spectra data that do not touch the sample, and ability to get different samples properties information in a single measurement that is related to their chemical and textural properties since spectroscopy covers from UV to NIR range. In addition, spectroscopy also has high reproducibility and good long-term stability (Abu-Khalaf, 2015). UV spectroscopy is used to identify the relative purity of a solution, which is quantifying protein, determining DNA concentration, and determining the ratio of protein to DNA concentration in a solution. Correspondingly, infrared (IR) spectroscopy can quantify different types of interatomic bond vibrations at different frequencies, such as analysing constituents like pigments and fillers, whereas near-infrared (NIR) spectroscopy is vital for practical applications since it has greater penetration depth into the sample.

The spectroscopy may consist of the spectroscopy with VIS/NIR wavelength. It is equipped with a fibre optic probe to acquire spectra data. Fibre optic probe delivers the light to the sample through fibres and collects it from the sample (Lorente et al., 2015). The spectroscopy system also consists of light source for spectra acquisition, a holder for locating the sample over the probe, and a computer equipped with commercial software for controlling the spectrometer and acquiring the spectra. The experimental set-up of the spectroscopy system is presented in Figure 3. This instrument measures the material absorption of the infrared radiation or transmittance. Certain wavelengths are absorbed, while the others are not as each substance has its own characteristic absorption spectrum. The instrument also can be applied to make a quantitative analysis of substances since the total amount of substances is proportional to the absorption of spectra. In addition, the general data systems of spectroscopy consist of a general computer connected to a printer that can produce high-quality spectra, as well as a software programme that includes spectrum acquisition offer, spectral transform, spectral processing, spectral analysis, and all other functions. Besides, users can also create their own operational procedures of spectral data for their needs (Tan et al., 2012).

Apart from that, principal component analysis (PCA) was initially performed in order to explore the data for any clustering (Finzi et al., 2015). PCA is a non-supervised linear multivariate technique for generating a new set of noncorrelated variables. It uses a mathematical procedure to transform a set of correlated response variables into principal components (PCs) (Abu-Khalaf, 2015). The PCs then represent a pattern of observation in plots. Therefore, the purpose of PCA is to show a linear relationship between different samples and variables, as well as the possibility of classifying the treatments during the experiment. The processed data were analysed by using partial least squares (PLS) to divide the spectra into subgroups and to study the correlation between the dependent and the independent variables in both calibration and crossvalidation tests. PLS is one of the regression algorithms employed in the chemometrics fields and built a linear relationship between independent and dependent variables.

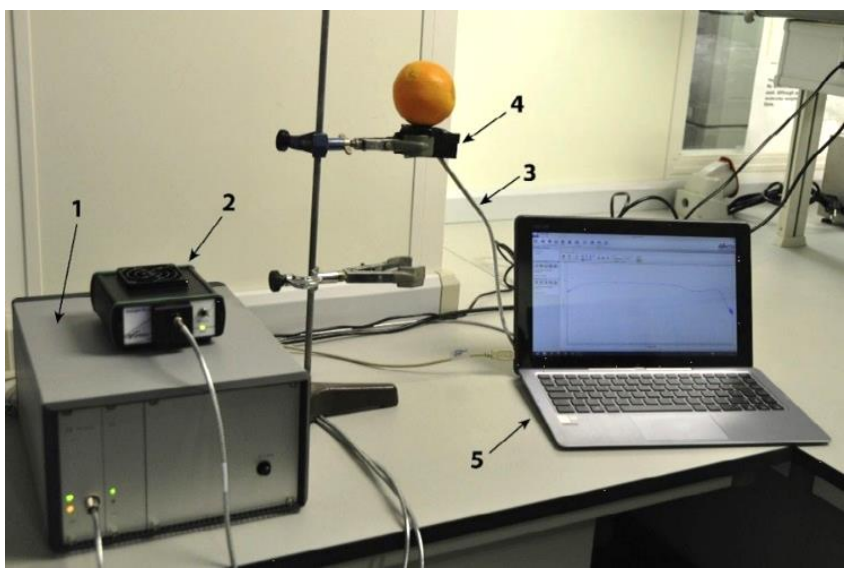

Figure 3. The experimental set-up for spectroscopy system: (1) Spectrophotometer platform, (2) Light source, (3) Reflectance probe, (4) Sample holder, (5) Computer with acquisition software (Lorente et al., 2015)

PLS estimates dependent variables as projections of the original input variables of independent and uses them to construct the regression vector in relation to independent and dependent variables. The results are evaluated by the performances of the coefficient of determination $\left(\mathrm{R}^{2}\right)$, the Root Mean Square in Cross-Validation (RMSECV), the ratio of performance to deviation (RPD), the slope of the regression line, the average accuracy of prediction that should be as close to 1 as possible, and the offset that should be as close to 0 as possible. The other recommended classifiers for classification are linear discriminant analysis (LDA), Naïve-Bayes (NB) classifier, quadratic discriminant analysis (QDA), and bagged decision tree (BDT) (Haff et al., 2014)

The spectroscopy technique has been widely used for many applications in agricultural product, especially for quality and properties evaluation, as well as disease or insect damage. These applications include the effect of measurement technique and sample preparation on estimation of total solids (TS), total kjeldahl nitrogen (TNK), total ammoniacal nitrogen (TAN), and volatile fatty acids (VFAs) in dairy and pig slurry, as well as in digestate by using Near-infrared spectroscopy (NIRS), as suggested by Finzi et al. (2015). The results indicated that filtered and homogenised samples displayed higher correlation $\left(\mathrm{R}^{2}\right)$ and the ratio of standard error of performance to standard deviation (RPD) had been $0.79<\mathrm{R}^{2}>0.98$ and $2.26<\mathrm{RPD}>6.99$ for filtered samples, while $0.30<\mathrm{R}^{2}>0.97$ and $1.24<\mathrm{RPD}>6.31$ for homogenised samples. Sankaran et al. (2012) performed an evaluation on classification between laurel wilt and freeze damage (leaf necrosis) from symptomatic and asymptomatic avocado leaves by using the VIS-NIR spectroscopy. The results revealed that the VIS-NIR spectroscopy could classify asymptomatic leaves from infected plants with $94 \%$ accuracies via QDA, BDT, linear discriminant analysis, and Naïve-Bayes classifiers. On top of that, the detection of olive fruit infested by Bactrocera oleae by using NIR spectroscopy was carried out by Haff et al. (2014). The results showed that the detection of hidden insect damage exhibited classification error rates as low as $0.00 \%$ false negative, $12.50 \%$ false negative, and $6.25 \%$ total error. Furthermore, there is a wide application of NIR spectroscopy on detection of insect damage because insects and larvae can be detected indirectly through their internal browning or darkening, fungi contamination or dehydration, or directly due to their hemolymph, chitin or lipid content.

The infrared spectroscopy is a flexible experiment technique and comparatively convenient to obtain spectra from samples. A spectrometer that has been predominantly used nowadays is the Fourier-transform infrared spectrometer (FTIR). FTIR spectroscopy yields an interferogram as the result of the distraction of radiation between two beams. Change of 
path length between two beams and two domains of frequency produces a signal. The distance is interconvertible through the mathematical method of Fourier-transformation. The basic components of FTIR spectrometer consist of a source, interferometer, sample, detector, amplifier, analogue-to-digital converter, and computer. Radiation produced by the FTIR spectrometer emerges from the source. It passes through the interferometer and the sample before reaching a detector. Then, the signal from the detector is amplified by an amplifier. Afterwards, the analogue-to-digital converter converts the data into digital form and the digital form is transferred to the computer for Fourier-transformation. The strength of FTIR is its speed advantage which makes it possible to obtain spectra on a millisecond timescale.

Tan et al. (2012) employed the NIR spectroscopy for detection of cold rice blast in cold land, which consisted of the healthy rice plant, three diseased levels of leaves blast, five diseased levels of grain blast, and four diseased levels of the infected stem. The study adopted the multi-spectral vision technology to investigate the identified classification test on the rice varieties, which had been resistance to disease. It is the combination of spectral analysis and image processing technology. The results showed that transmittance of the spectrum of different parts of the rice plant, which were stems, leaves, and grains, were not in the same position. Leaves and grain transmittance spectra had almost similar intensity, while stems transmission had been relatively low. In addition, disease transmission in different degrees of intensity became less evident in the diseased leaves when the wavelength increased close to $4000 \mathrm{~nm}$. The results revealed that under different conditions, different plant parts had their own characteristics in NIR spectral bands. Moreover, the regularity of the stems and the grains had been better than that of leaves. The differences in grains had been obvious in the $7000 \mathrm{~nm}-10000 \mathrm{~nm}$ wavelengths, while $4000-7000 \mathrm{~nm}$ for stems. These may be due to the slight changes that took place in both the stem and the seed during growth, whereas the leaves were affected as they had insufficient water.

\subsection{Remote Sensing}

Identification of earth features through the detection of electromagnetic radiation characteristic that is emitted or reflected by the earth surface is known as Remote Sensing (RS). The radiation that is received from the target in different spectral regions is detected by the sensors onboard on various platforms. It is an effective measurement method and inexpensive (Christensen et al., 2004). The measurement of disease incidence for many agricultural crops has been carried out by using aerial photography, satellite remote sensing, airborne sensor data, ground-based sensor data, and hyperspectral remote sensing. Aerial photography is the technique of taking images of the ground from an elevated position and the images are mounted on aerial vehicles (UAV). Aerial photography is low-altitude remote sensing (LARS), which can acquire images with higher resolution and lower cost. The drawback of the aerial photograph is that it is affected by large or numerous patches of exposed soil in a field, which could lead to interference with disease interpretation. Besides, diseased leaves that were covered by the upper canopy of healthy green foliage cannot be detected by using the aerial photograph.

Satellite remote sensing is a technique of acquisition of images for large field area. Rush et al. (2010) carried out a study on detecting and quantifying wheat infected by wheat streak mosaic virus by using Landsat 5 Thematic Mapper (TM). The results showed that Landsat $5 \mathrm{TM}$ had managed to classify healthy and diseased wheat with accuracies between 89.47 and $99.07 \%$. However, satellite remote sensing can be affected by weather conditions, have long revisit time and inappropriate for small scale field when it involves low-resolution satellite images. Meanwhile, the airborne sensor data is a technique of remote sensing that uses platforms and sensors to acquire images of the ground. Some of the platforms of airborne are airship, UAV, helicopter, and aircraft. The advantage of airborne remote sensing over satellite is that the user can define the deployment and the operational characteristics of the remote sensing. It also can provide higher spatial resolution data, rapid image acquisition, adjustable spatial resolutions at different flight altitudes, and adjustment of speed image acquisition. In fact, Qin and Zhang (2005) conducted a study on detection of rice sheath blight by using high spatial resolution ADAR (Airborne Data Acquisition and Registration) remote sensing. The results showed that airborne remote sensing could be applied for the detection of rice sheath blight disease, however, identification of infection increased when infection of disease was between medium and severe levels.

On the other hand, the ground-based sensor data is a kind of remote sensing that uses ground-based platforms, such as spectroradiometer or portable field spectrometer. For ground truth measurements, Zhu et al. (2011) showed that groundbased remote sensing can be used to identify and estimate rice neck severity, as well as rice infested by leaf folder with $\mathrm{R}^{2}=$ 0.827. However, the spectral response on the crop depends on atmospheric conditions, such as illumination and cloudy shadow; biotic, such as leaf area index; and edaphic, such as soil type and moisture. Meanwhile, the hyperspectral remote sensing is a technique that operates in hundreds of narrow wavelength bands by utilizing sensors with potential to improve the performance of assessment of crop diseases. Zhou et al. (2007) estimated rice brown spot disease by using hyperspectral remote sensing. The study proved that it was feasible to estimate rice brown spot disease severity with RMSE of training and testing of $4.1 \%$ and $2.0 \%$ of PLS regression analysis.

Basically, the imaging system for RS has a few essential components, which are energy source or illumination, sensors, surface targets, and atmospheric effects. The energy source (A) provides electromagnetic energy to the target region. It is natural as it originates from the sun or the earth by emission. The energy interacts with the atmosphere (B) it passes through while travelling from the source to the target. The interaction depends on the properties of both the target and the radiation. The radiation may be transmitted, absorbed, emitted, scattered or reflected by a target substance. After the energy has been reflected or absorbed by the target, a sensor (D) collects and records the electromagnetic radiation. Sensors are classified based on the energy sources used, which may be passive or active sensors. Sensors that sense natural radiations, which are reflected or emitted from the earth, are known as passive sensors; while sensors that produce their own electromagnetic radiation are known as active sensors. Most RS sensors are passive as they measure the solar radiation reflected from the target region. Since RS is classified as optical and microwave, sensors that detect solar radiation in the visible, near-infrared, middle-infrared, and thermal-infrared wavelength regions are known as optical RS. Meanwhile, sensors that work in the region of electromagnetic waves with frequencies between 109 and $1012 \mathrm{~Hz}$ are microwave RS. Sensors for observations need to be mounted on a platform that can be classified as ground-based, such as handheld radiometers, airborne such as AVIRIS sensor of NASA, and spaceborne such as satellite-based.

The application of RS in monitoring pest and disease on rice is summarized in Table 2. To date, many studies have detected rice diseases, such as rice brown spot, bacterial leaf blight, sheath blight, rice panicle blast, rice neck blast, and rice false smut. Airborne multispectral scanning has been widely applied to examine the spectral response of canopies of the diseased 
plant for surveillance of environmental stresses, pests and plant diseases, as well as ground-based radiometry (Kanda et al., 2007). The feasibility of discriminating diseases of crop fields from the healthy crop has been carried out by using multispectral remote sensing data that are available from spacebased and airborne sensors (Singh et al., 2012). Space-based sensors usually provide reflectance that consists of a few selected wavelength regions called bands at around the bandwidth of $60-80 \mathrm{~nm}$. The advantages of using the satellite RS are that it is potential for fast survey, low in cost, higher accuracy, use of multispectral data for increased information, inaccessible area coverage, capability at all weathers, day or night, as well as the capability of performing simultaneous observations from a single platform at different resolutions or angles. Presently, the hyperspectral RS has been used for the detection of plant disease. It utilizes sensors and operates in hundreds of narrow wavelength bands of $10 \mathrm{~nm}$ or less. Narrow bands are better than a few selected wavelengths as they can measure the exact characteristic of absorption peaks of plant pigments, which lead to more accurate information concerning plant health.

Hyperspectral remote sensing provides additional bands in the visible, NIR, and shortwave-infrared (SWIR) regions (Prasannakumar et al., 2013). Its sensors acquire radiance information from the visible region to the SWIR region (400$2500 \mathrm{~nm}$ ) in less than $10 \mathrm{~nm}$. The application of hyperspectral remote sensing enables the collection of several hundreds of spectral bands in a single acquisition, which leads to the production of more detailed spectral data than broadband technique, with the potential to improve the assessment of crop disease. Zhu et al. (2011) estimated the rice neck blasts severity by using spectral reflectance and constructed artificial neural network model (BP model) for the rice neck blast modelling. The severity of diseases among the infected plants was then measured based on the percentage of infection of the fringes. Besides, diseases index (DI) was used to classify the infected fringes into six grades $(0,1,2,3,4$, and 5$)$. The results showed that in the visible $(400-745 \mathrm{~nm})$ and the NIR $(805-1000 \mathrm{~nm})$ spectral regions, $\mathrm{DI}=3$ with moderate neck blast showcased high raw reflectance, while the visible $(750-800 \mathrm{~nm})$ spectral region displayed low raw reflectance. In addition, $\mathrm{DI}=5$, which referred to rice fringes with serious neck blast, portrayed high reflectance at NIR $(960-1000 \mathrm{~nm})$ spectral regions, while at wavelength 400 to $960 \mathrm{~nm}$, low raw reflectance was noted (Figure 4). The differences of spectral reading showed some changes in the cellular structure and the content of the pigment on the infected rice neck. In the visible $(520-550 \mathrm{~nm})$ spectral regions, serious neck blast had low FDR, while at a wavelength of 710-750 nm spectral region; moderate neck blast had low FDR. Next, BP-neutral network was constructed and provided better accuracy in the retrieval of DI compared to a regression model with RMSE, $\mathrm{R}^{2}$ and CRR of $0.084,0.992$, and $100 \%$.

Characterization of brown planthopper (BPH) damage on rice plants through hyperspectral remote sensing under field conditions had been carried out by Prasannakumar et al. (2014). The study discovered that in the VIS region $(400-700 \mathrm{~nm})$, spectral reflectance of uninfected plants was lower than infested crops, while reflectance of the infested plant increased with the increase in damage. Figure 5 shows peaks that were revealed when values of correlation coefficients were plotted against wavelength, whereby four sensitive wavebands were identified; $764 \mathrm{~nm}(\mathrm{r}=0.674), 961 \mathrm{~nm}(\mathrm{r}=0.70), 1201 \mathrm{~nm}(\mathrm{r}=0.70)$, and $1664 \mathrm{~nm}(\mathrm{r}=0.60)$. Then, three new BPH indices were formulated

Table 2. Studies pertaining to rice diseases detection by using remote sensing application

\begin{tabular}{|c|c|c|c|c|}
\hline Types of systems & $\begin{array}{l}\text { Types of paddy } \\
\text { diseases }\end{array}$ & $\begin{array}{l}\text { Wavelength } \\
\text { range }\end{array}$ & Significant wavelength & References \\
\hline Spectrophotometer & $\begin{array}{l}\text { Rice false smut, rice } \\
\text { glume blight }\end{array}$ & $350-2500 \mathrm{~nm}$ & $680,750,695,694,735 \mathrm{~nm}$ & Liu et al. (2010) \\
\hline Spectroradiometer & Bacterial leaf blight & $330-2600 \mathrm{~nm}$ & 757-1039nm & Yang (2010) \\
\hline Spectroradiometer & Rice neck blast & $400-1000 \mathrm{~nm}$ & $685,711 \mathrm{~nm}$ & Zhu et al. (2011) \\
\hline Spectroradiometer & Rice leaf blight & $325-1075 \mathrm{~nm}$ & $770-860 \mathrm{~nm}, 920-1050 \mathrm{~nm}$ & Singh et al. (2012) \\
\hline $\begin{array}{l}\text { ASD FieldSpec } \\
\text { Spectrometer }\end{array}$ & Brown spot & $350-2500 \mathrm{~nm}$ & $401-530,550-730 \mathrm{~nm}$ & Zhao et al. (2012) \\
\hline Spectroradiometer & Rice infested by BPH & $350-2500 \mathrm{~nm}$ & $500,665,1792,1986 \mathrm{~nm}$ & $\begin{array}{l}\text { Prasannakumar et al. } \\
\text { (2013) }\end{array}$ \\
\hline Spectroradiometer & Rice infested by BPH & $350-2500 \mathrm{~nm}$ & $764,961,1201,1664 \mathrm{~nm}$ & $\begin{array}{l}\text { Prasannakumar and } \\
\text { Chander (2013) }\end{array}$ \\
\hline Spectroradiometer & Rice infested by BPH & $325-107 \mathrm{~nm}$ & - & Huang et al. (2015) \\
\hline SPOT-5 images & $\mathrm{BPH}$ & $530-2500 \mathrm{~nm}$ & $\begin{array}{l}530-600 \mathrm{~nm}, 610-680 \mathrm{~nm}, 780- \\
1000 \mathrm{~nm}, 1000-2500 \mathrm{~nm}\end{array}$ & Ghobadifar et al. (2014) \\
\hline $\begin{array}{l}\text { hyperspectral and LISS IV } \\
\text { satellite }\end{array}$ & Bacterial leaf blight & $350-2500 \mathrm{~nm}$ & $760,990,680$ and $540 \mathrm{~nm}$ & Das et al. (2015) \\
\hline $\begin{array}{l}\text { Airborne hyperspectral } \\
\text { imagery }\end{array}$ & Rice blast & $430-1000 \mathrm{~nm}$ & $498-515 \mathrm{~nm}, 700-717 \mathrm{~nm}$ & Kobayashi et al. (2016) \\
\hline $\begin{array}{l}\text { Multispectral remote } \\
\text { sensing }\end{array}$ & $\mathrm{BPH}$ & $530-2500 \mathrm{~nm}$ & $\begin{array}{l}530-600 \mathrm{~nm}, 610-680 \mathrm{~nm}, 780- \\
1000 \mathrm{~nm}, 1000-2500 \mathrm{~nm}\end{array}$ & Ghobadifar et al. (2016) \\
\hline PlanetScope & $\begin{array}{l}\text { rice dwarf, rice blast, } \\
\text { glume blight }\end{array}$ & $450-1000 \mathrm{~nm}$ & $\begin{array}{l}455-515 \mathrm{~nm}, 500-590 \mathrm{~nm}, 590- \\
670 \mathrm{~nm}, 780-860 \mathrm{~nm}\end{array}$ & Shi et al. (2018) \\
\hline Landsat-8 images & Rice sheath blight & - & - & Zhang et al. (2018) \\
\hline $\begin{array}{l}\text { Hyperspectral remote } \\
\text { sensing }\end{array}$ & Rice blast & $360-1025 \mathrm{~nm}$ & $450-800 \mathrm{~nm}$ & Tian et al. (2018) \\
\hline Spectrophotometer & $\begin{array}{l}\text { Rice foliar disease } \\
\text { and insect damage }\end{array}$ & $350-2500 \mathrm{~nm}$ & $\begin{array}{l}400-720 \mathrm{~nm}, 720-1200 \mathrm{~nm}, \\
1200-2400 \mathrm{~nm}\end{array}$ & Liu et al. (2018) \\
\hline
\end{tabular}

BPH, brown plant hopper; LISS, linear imaging self-scanner. 

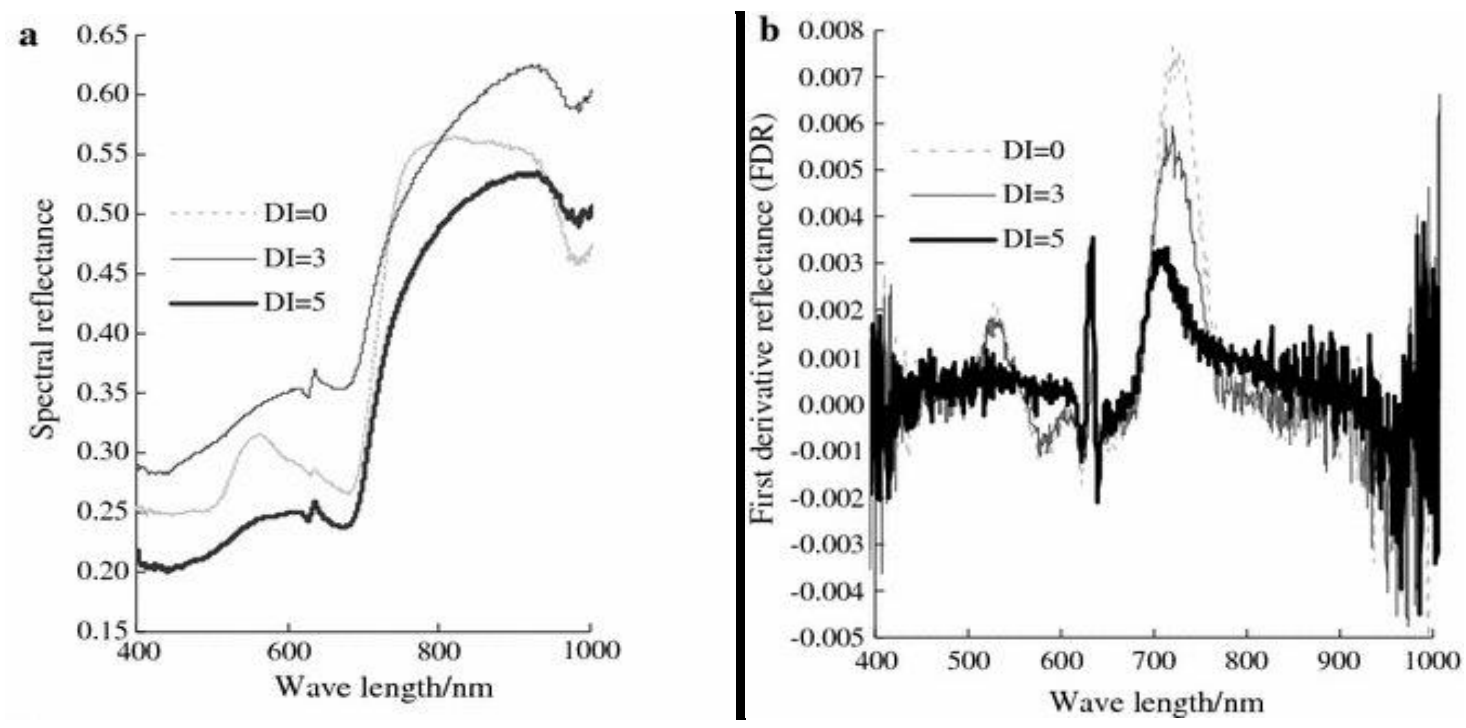

Figure 4. Reflectance spectra: (a) and first derivative reflectance, (b) of DI=5 (serious), DI=3 (moderate) and DI=0 (healthy) (Zhu et al., 2011)

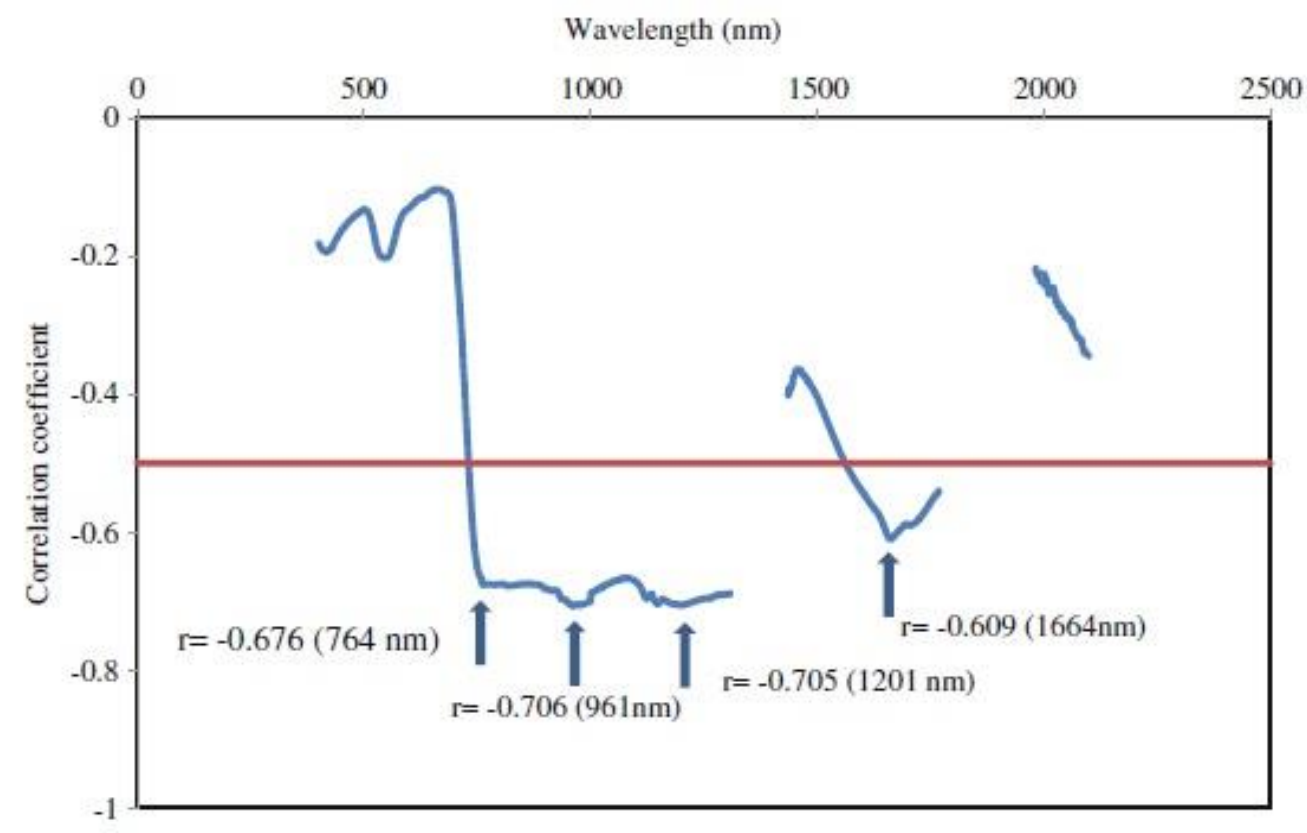

Figure 5. Infested rice crop reflectance of different BPH damage levels at $1 \mathrm{~nm}$ interval against values of correlation coefficients (Prasannakumar et al., 2014)

by using two or more sensitive wavebands, which had been brown planthopper index-1 (BPHI-1), brown planthopper index-2 (BPHI-2), and brown planthopper index-3 (BPHI-3), that showed a good relationship with BPH infestation levels. Furthermore, the BPH damage reflectance model, which is a multilinear regression model, was developed between damage levels and plant reflectance with $\mathrm{R}^{2}=0.71$ and $\mathrm{RMSE}=1.74$, while validation with $\mathrm{R}^{2}=0.73$ and $\mathrm{RMSE}=0.71$ accounted for $73 \%$ of the variability in the BPH damage.

\section{Conclusions}

This paper presents a review on the optical imaging techniques, which are non-destructive techniques that have been used for rice disease detection. The imaging-based techniques include computer vision, spectroscopic, HSI, and remote sensing. The HSI and remote sensing approaches have been more preferred as they operate in narrow wavelength bands, which are better than from a few selected wavelengths, as narrow wavelength can measure the exact characteristic of the absorption peaks of plant pigment. Moreover, the measurement of this exact characteristic can lead to more accurate information concerning plant health, as well as diseases. However, HSI has some drawbacks pertaining to the volume of data, noises from the sensor, calibration errors, and shot errors that limit the interpretation, as well as the analysis of the images. Meanwhile, applications of remote sensing are good for large scale farm, but the ADAR fails in differentiating between very low infection and healthy plants. The application of remote sensing at the canopy level depends on several factors, such as atmospheric like cloud shadow and illumination; edaphic like soil type and moisture; as well as biotic like crop variety, leaf area index, plant architecture, wind, and field management. The review portrays that these techniques of rice disease detection possess the ability to rapidly and accurately detect rice diseases. Therefore, some improvements should be 
made to overcome the limitations of each technique. The image processing and imaging-based techniques could be integrated with the agricultural vehicle for on-site rice disease detection for the purposes of disease control, prevention, and management. Nevertheless, in the computer vision technique, a few things must be noted to obtain a high rate of accuracy, for instance, selection of features to be extracted, image pre-processing steps to get noise-free image, selection of camera to get good quality image for better segmentation, and determination of threshold value to avoid error during the classification phase. Recent works provide the notion that optical imaging techniques can be used for rice diseases detection. The changes from the conventional approach to the modern techniques indicated that the commercialized industries for rice crop need a robust method for monitoring the rice diseases at an early stage. One of the major challenges in the implementation of optical imaging techniques for rice disease detection is the configuration of the device in a suitable condition. Hence, it is possible to integrate the optical imaging into a robust model so that these approaches are developed for industrial purposes. The advantages of these techniques demonstrated the detection of rice diseases apart from improving rice growth and minimizing the problems of the disease. With the advancement of optical imaging technologies, the requirement for rice yield needed will be achieved along with further research and progression.

\section{Conflict of interests}

The authors have declared no conflict of interest.

\section{Author contributions}

Nur Azizah Bachik performed the research works and drafted the manuscript. Norhashila Hashim supervised and reviewed the manuscript. Aimrun Wayayok and Hasfalina Che Man gave critical comments and suggestions related to the study. Maimunah Mohd Ali finalised the whole manuscript.

\section{Acknowledgments}

The authors wish to acknowledge the Department of Biological and Agricultural Engineering, Faculty of Engineering, Universiti Putra Malaysia for supporting this research under Putra Grant (GP-IPB) (Vot. No.:9442500).

\section{References}

Abu-Khalaf, N. (2015). Sensing tomato's pathogen using Visible/Near infrared (VIS/NIR) spectroscopy and multivariate data analysis (MVDA). Palestine Technical University Research Journal, 3(1), 12-22.

Abu Bakar, M. N., Abdullah, A. H., Rahim, N. A., Yazid, H., Misman, S. N., \& Masnan, M. J. (2018). Rice Leaf Blast Disease Detection Using Multi-Level Colour Image Thresholding. Journal of Telecommunication, Electronic and Computer Engineering, 10(1), 1-6.

Adebayo, S. E., Hashim, N., Abdan, K., \& Hanafi, M. (2016). Application and potential of backscattering imaging techniques in agricultural and food processing - A review. Journal of Food Engineering, 169, 155-164.

Aleixos, N., Blasco, J., Navarrón, F., \& Moltó, E. (2002). Multispectral inspection of citrus in real-time using machine vision and digital signal processors. Computers and Electronics in Agriculture, 33, 121-137.

Ali, M. M., Bachik, N. A., Muhadi, N. 'Atirah, Tuan Yusof, T. N., \& Gomes, C. (2019). Non-destructive techniques of detecting plant diseases: A review. Physiological and Molecular Plant Pathology, 108, 1-12.
Asfarian, A., Herdiyeni, Y., Rauf, A., \& Mutaqin, K. H. (2013). Paddy diseases identification with texture analysis using fractal descriptors based on fourier spectrum. Proceeding 2013 International Conference on Computer, Control, Informatics and Its Applications: "Recent Challenges in Computer, Control and Informatics", IC3INA 2013, 77-81.

Baranowski, P., Mazurek, W., Wozniak, J., \& Majewska, U. (2012). Detection of early bruises in apples using hyperspectral data and thermal imaging. Journal of Food Engineering, 110(3), 345-355.

Bauriegel, E., \& Herppich, W. (2014). Hyperspectral and Chlorophyll Fluorescence Imaging for Early Detection of Plant Diseases, with Special Reference to Fusarium spec. Infections on Wheat. Agriculture, 4(1), 32-57.

Brosnan, T., \& Sun, D. W. (2004). Improving quality inspection of food products by computer vision - A review. Journal of Food Engineering, 61, 3-16.

Buschmann, C., Lenk, S., \& Lichtenthaler, H. K. (2012). Reflectance spectra and images of green leaves with different tissue structure and chlorophyll content. Israel Journal of Plant Sciences, 60(1), 49-64.

Casanova, J. J., O'Shaughnessy, S. A., Evett, S. R., \& Rush, C. M. (2014). Development of a wireless computer vision instrument to detect biotic stress in wheat. Sensors (Switzerland), 14(9), 17753-17769.

Chen, X., Ke, S., Wang, L., Xu, H., \& Chen, W. (2012). Classification of rice appearance quality based on LS-SVM using machine vision. Communications in Computer and Information Science, 307 CCIS (PART 1), 104-109.

Christensen, T., Minghua Zhang, Wenjuan Li, Huajun Tang, \& Zhihao Qin. (2004). Remote sensing analysis of rice disease stresses for farm pest management using wide-band airborne data. 00(C), 2215-2217.

Chung, C. L., Huang, K. J., Chen, S. Y., Lai, M. H., Chen, Y. C., \& Kuo, Y. F. (2016). Detecting Bakanae disease in rice seedlings by machine vision. Computers and Electronics in Agriculture, $121,404-411$.

Das, P. K., Laxman, B., Rao, S. V. C. K., Seshasai, M. V. R., \& Dadhwal, V. K. (2015). Monitoring of bacterial leaf blight in rice using ground-based hyperspectral and LISS IV satellite data in Kurnool, Andhra Pradesh, India. International Journal of Pest Management, 61(4), 359-368.

El Masry, G., Sun, D. W., \& Allen, P. (2011). Non-destructive determination of water-holding capacity in fresh beef by using NIR hyperspectral imaging. Food Research International, 44(9), 2624-2633.

El Masry, G., Wang, N., ElSayed, A., \& Ngadi, M. (2007). Hyperspectral imaging for nondestructive determination of some quality attributes for strawberry. Journal of Food Engineering, 81(1), 98-107.

Finzi, A., Oberti, R., Negri, A. S., Perazzolo, F., Cocolo, G., Tambone, F., Cabassi, G., \& Provolo, G. (2015). Effects of measurement technique and sample preparation on NIR spectroscopy analysis of livestock slurry and digestates. Biosystems Engineering, 134, 42-54.

Garrido-Novell, C., Pérez-Marin, D., Amigo, J. M., FernándezNovales, J., Guerrero, J. E., \& Garrido-Varo, A. (2012). Grading and color evolution of apples using RGB and hyperspectral imaging vision cameras. Journal of Food Engineering, 113(2), 281-288.

Ghobadifar, F., Wayayok, A., Shattri, M., \& Shafri, H. (2014). Using SPOT-5 images in rice farming for detecting BPH (Brown Plant Hopper). IOP Conference Series: Earth and Environmental Science, 20(1), 1-10.

Ghobadifar, Faranak, Aimrun, W., \& Jebur, M. N. (2016). Development of an early warning system for brown planthopper (BPH) (Nilaparvata lugens) in rice farming using multispectral remote sensing. Precision Agriculture, 17(4), 377-391. 
Ghyar, B. S., \& Birajdar, G. K. (2017). Computer vision based approach to detect rice leaf diseases using texture and color descriptors. Proceedings of the International Conference on Inventive Computing and Informatics, ICICI 2017, 10741078.

Haff, R. P., Moscetti, R., Monarca, D., Massantini, R., Cecchini, M., Contini, M., \& Stella, E. (2014). Feasibility of NIR spectroscopy to detect olive fruit infested by Bactrocera oleae. Postharvest Biology and Technology, 99(July), 58-62.

Hashim, N., Pflanz, M., Regen, C., Janius, R. B., Abdul Rahman, R., Osman, A., Shitan, M., \& Zude, M. (2013). An approach for monitoring the chilling injury appearance in bananas by means of backscattering imaging. Journal of Food Engineering, 116(1), 28-36.

Hongwei, Y., Ken, C., Hanhui, L., Zhihui, C., \& Zhaofeng, Z. (2016). Segmentation of rice planthoppers in rice fields based on an improved level-set approach. INMATEH - Agricultural Engineering, 48(1), 67-74.

Hu, H., Liu, H. Q., Zhang, H., Zhu, J. H., Yao, X. G., Zhang, X. Bin, \& Zheng, K. F. (2010). Assessment of chlorophyll content based on image color analysis, comparison with SPAD-502. 2nd International Conference on Information Engineering and Computer Science - Proceedings, ICIECS 2010, 1-3.

Huang, J., Sun, J., Liao, H., \& Liu, X. (2015). Detection of brown planthopper infestation based on SPAD and spectral data from rice under different rates of nitrogen fertilizer. Precision Agricultural, 16, 148-163.

Huang, S., Qi, L., Ma, X., Xue, K., Wang, W., \& Zhu, X. (2015). Hyperspectral image analysis based on BoSW model for rice panicle blast grading. Computers and Electronics in Agriculture, 118, 167-178.

Kanda, E., Torigoe, Y., Kitada, K., Ishiguro, K., \& Kobayashi, T. (2007). Detection of Rice Panicle Blast with Multispectral Radiometer and the Potential of Using Airborne Multispectral Scanners. Phytopathology, 91(3), 316-323.

Kobayashi, T., Kanda, E., Kitada, K., Ishiguro, K., \& Torigoe, Y. (2007). Detection of Rice Panicle Blast with Multispectral Radiometer and the Potential of Using Airborne Multispectral Scanners. Phytopathology, 91(3), 316-323.

Kobayashi, Takashi, Sasahara, M., Kanda, E., Ishiguro, K., Hase, S., \& Torigoe, Y. (2016). Assessment of Rice Panicle Blast Disease Using Airborne Hyperspectral Imagery. The Open Agriculture Journal, 10(1), 28-34.

Kurniawati, N. N., Abdullah, S. N. H. S., Abdullah, S., \& Abdullah, S. (2009). Investigation on Image Processing Techniques for Diagnosing Paddy Diseases. 2009 International Conference of Soft Computing and Pattern Recognition, 272-277.

Liu, C., Liu, W., Chen, W., Yang, J., \& Zheng, L. (2015). Feasibility in multispectral imaging for predicting the content of bioactive compounds in intact tomato fruit. Food Chemistry, $173,482-488$.

Liu, C., Liu, W., Lu, X., Chen, W., Yang, J., \& Zheng, L. (2014). Nondestructive determination of transgenic Bacillus thuringiensis rice seeds (Oryza sativa L.) using multispectral imaging and chemometric methods. Food Chemistry, 153, 87-93.

Liu, Z.-Y., Wu, H.-F., \& Huang, J.-F. (2010). Application of neural networks to discriminate fungal infection levels in rice panicles using hyperspectral reflectance and principal components analysis. Computers and Electronics in Agriculture, 72(2), 99-106.

Liu, Z., Shi, J., Zhang, L., \& Huang, J. (2010). Discrimination of rice panicles by hyperspectral reflectance data based on principal component analysis and support vector classification. Journal of Zhejiang University SCIENCE B, 11(1), 71-78.

Liu, Z. Y., Qi, J. G., Wang, N. N., Zhu, Z. R., Luo, J., Liu, L. J., Tang, J., \& Cheng, J. A. (2018). Hyperspectral discrimination of foliar biotic damages in rice using principal component analysis and probabilistic neural network. Precision Agriculture, 19(6), 973-991.

Lorente, D., Blasco, J., Serrano, A. J., Soria-Olivas, E., Aleixos, N., \& Gómez-Sanchis, J. (2013). Comparison of ROC Feature Selection Method for the Detection of Decay in Citrus Fruit Using Hyperspectral Images. Food and Bioprocess Technology, 6(12), 3613-3619.

Lorente, D., Escandell-Montero, P., Cubero, S., Gómez-Sanchis, J., \& Blasco, J. (2015). Visible-NIR reflectance spectroscopy and manifold learning methods applied to the detection of fungal infections on citrus fruit. Journal of Food Engineering, 163, 17-24.

Lu, R. (2004). Multispectral imaging for predicting firmness and soluble solids content of apple fruit. Postharvest Biology and Technology, 31(2), 147-157.

Mahesh, S., Jayas, D. S., Paliwal, J., \& White, N. D. G. (2015). Hyperspectral imaging to classify and monitor quality of agricultural materials. Journal of Stored Products Research, 61, 17-26.

Maheshwari, C. V. (2013). Machine Vision Technology for Oryza Sativa L. (Rice). International Journal of Advanced Research in Electrical, Electronics and Instrumentation Engineering, 2(7), 2893-2900.

Mohd Ali, M., Hashim, N., Bejo, S. K., \& Shamsudin, R. (2020). Comparison of laser backscattering imaging and computer vision system for grading of seedless watermelons. Journal of Food Measurement and Characterization, 14, 69-77.

Narendra, V. G., \& Hareesh, K. S. (2010). Quality Inspection and Grading of Agricultural and Food Products by Computer Vision-a Review. International Journal of Computer Applications, 2(1), 43-65.

Patel, K. K., Kar, A., Jha, S. N., \& Khan, M. A. (2012). Machine vision system: A tool for quality inspection of food and agricultural products. Journal of Food Science and Technology, 49(2), 123-141.

Pereira, F. M. V., Milori, D. M. B. P., Venâncio, A. L., Russo, M. D. S. T., Martins, P. K., \& Freitas-Astúa, J. (2010). Evaluation of the effects of Candidatus Liberibacter asiaticus on inoculated citrus plants using laser-induced breakdown spectroscopy (LIBS) and chemometrics tools. Talanta, 83(2), 351-356.

Phadikar, S., \& Sil, J. (2008). Rice disease identification using pattern recognition techniques. Computer and Information Technology, Iccit, 420-423.

Phadikar, S., Sil, J., \& Das, A. K. (2013). Rice diseases classification using feature selection and rule generation techniques. Computers and Electronics in Agriculture, 90, 76-85.

Prasannakumar, N., \& Chander, S. (2013). Spectral signatures of rice crop damaged by brown planthopper under field and glass house conditions. Current Biotica, $7(3), 124-133$

Prasannakumar, N. R., Chander, S., \& Sahoo, R. N. (2014). Characterization of brown planthopper damage on rice crops through hyperspectral remote sensing under field conditions. Phytoparasitica, 42(3), 387-395.

Prasannakumar, N. R., Chander, S., Sahoo, R. N., \& Gupta, V. K. (2013). Assessment of Brown Planthopper, (Nilaparvata lugens) [Stål], damage in rice using hyperspectral remote sensing. International Journal of Pest Management, 59(3), 180-188.

Qin, J., Burks, T. F., Ritenour, M. A., \& Bonn, W. G. (2009). Detection of citrus canker using hyperspectral reflectance imaging with spectral information divergence. Journal of Food Engineering, 93(2), 183-191.

Qin, Z., \& Zhang, M. (2005). Detection of rice sheath blight for inseason disease management using multispectral remote sensing. International Journal of Applied Earth Observation and Geoinformation, 7(2), 115-128.

Renugambal, K., \& Senthilraja, B. (2015). Application of Image Processing Techniques in Plant Disease Recognition. 
International Journal of Engineering Research \& Technology, 4(3), 919-923.

Rush, C. M., Mirik, M., Ansley, R. J., Jones, D. C., Workneh, F., \& Price, J. A. (2010). Satellite Remote Sensing of Wheat Infected by Wheat streak mosaic virus. Plant Disease, 95(1), 4-12.

Sankaran, S., Ehsani, R., Inch, S. A., \& Ploetz, R. C. (2012). Evaluation of Visible-Near Infrared Reflectance Spectra of Avocado Leaves as a Non-destructive Sensing Tool for Detection of Laurel Wilt. Plant Disease, 96(11), 1683-1689.

Shankar Ray, S. (2004). Basics of remote sensing. In Remote sensing image analysis: including the spatial domain (pp. 17).

Sharma, S., \& Dasgupta, I. (2012). Development of SYBR Green I based real-time PCR assays for quantitative detection of Rice tungro bacilliform virus and Rice tungro spherical virus. Journal of Virological Methods, 181(1), 86-92.

Shi, Y., Huang, W., Ye, H., Ruan, C., Xing, N., Geng, Y., Dong, Y., \& Peng, D. (2018). Partial least square discriminant analysis based on normalized two-stage vegetation indices for mapping damage from rice diseases using planetscope datasets. Sensors (Switzerland), 18(6), 1-16.

Shrestha, R., \& Hardeberg, J. Y. (2014). Evaluation and comparison of multispectral imaging systems. Final Program and Proceedings - IS and T/SID Color Imaging Conference, 107-112.

Singh, B., Singh, M., Singh, G., Suri, K., Pannu, P. P. S., \& Bal, S. K. (2012). Hyper-Spectral Data for the Detection of Rice Bacterial Leaf Blight (Blb) Disease. Agro-Informatics and Precision Agriculture, 177-182.

Steiner, U., Hillnhütter, C., Dehne, H.-W., Oerke, E.-C., \& Mahlein, A.-K. (2012). Hyperspectral imaging for small-scale analysis of symptoms caused by different sugar beet diseases. Plant Methods, 8(1), 3.

Suman, T., \& Dhruvakumar, T. (2015). Classification of paddy leaf diseases using shape and color features. International Journal of Electrical and Electronics Engineers, 7(1), 239250.

Surendrababu, V., Sumathi, C. P., \& Umapathy, E. (2014). Detection of Rice Leaf Diseases Using Chaos and Fractal Dimension in Image Processing. International Journal on Computer Science and Engineering, 6(01), 69-74.

Tan, F., Ma, X., Wang, C., \& Shang, T. (2012). Data Analysis of Cold Rice Blast Based on Near Infrared Spectroscopy. In D. Li \& Y. Chen (Eds.), Computer and Computing Technologies in Agriculture V (Vol. 369, pp. 64-71). Springer Berlin Heidelberg.

Tan, K. Z., Chai, Y. H., Song, W. X., \& Cao, X. Da. (2014). Identification of diseases for soybean seeds by computer vision applying BP neural network. International Journal of Agricultural and Biological Engineering, 7(3), 43-50.

Tanmayee, P. (2017). Rice crop monitoring system-A lot based machine vision approach. 2017 International Conference on Nextgen Electronic Technologies: Silicon to Software, ICNETS2 2017, 26-29.

Tian, L., Wan, Z., Li, D., Jiang, J., Yao, X., Cao, Q., Tian, Y., Zhu, Y., Cao, W., \& Cheng, T. (2018). Detecting rice blast disease using model inverted biochemical variables from closerange reflectance imagery of fresh leaves. International Geoscience and Remote Sensing Symposium (IGARSS), 2749-2752.

Valente, M., Leardi, R., Self, G., Luciano, G., \& Pain, J. P. (2009). Multivariate calibration of mango firmness using vis/NIR spectroscopy and acoustic impulse method. Journal of Food Engineering, 94(1), 7-13.
Williams, P., Geladi, P., Fox, G., \& Manley, M. (2009). Maize kernel hardness classification by near infrared (NIR) hyperspectral imaging and multivariate data analysis. Analytica Chimica Acta, 653, 121-130.

Yang, C. M. (2010). Assessment of the severity of bacterial leaf blight in rice using canopy hyperspectral reflectance. Precision Agriculture, 11(1), 61-81.

Yang, Y., Chai, R., \& He, Y. (2012). Early detection of rice blast (Pyricularia) at seedling stage in Nipponbare rice variety using near-infrared hyper-spectral image. African Journal of Biotechnology, 11(26), 6809-6817.

Yao, Q., Guan, Z., Zhou, Y., Tang, J., Hu, Y., \& Yang, B. (2009). Application of support vector machine for detecting rice diseases using shape and color texture features. 2009 International Conference on Engineering Computation, ICEC 2009, 79-83.

Zhang, D., Zhou, X., Zhang, J., Lan, Y., Xu, C., \& Liang, D. (2018). Detection of rice sheath blight using an unmanned aerial system with high-resolution color and multispectral imaging. PLoS ONE, 13(5), 1-14.

Zhang, X., Wang, B., Liu, P., Che, H., Zhou, X., \& Zhang, J. (2018). Influence of landscape pattern on epidemic of rice sheath blight disease at regional scale. 2018 7th International Conference on Agro-Geoinformatics, Agro-Geoinformatics 2018, 1-5.

Zhao, J., Zhang, D., Luo, J., Dong, Y., Yang, H., \& Huang, W. (2012). Characterization of the rice canopy infested with brown spot disease using field hyperspectral data. Wuhan University Journal of Natural Sciences, 17(1), 86-92.

Zhou, W., Shi, J., Huang, J., Zhang, L., Liu, Z., \& Tao, R. (2007). Characterizing and estimating rice brown spot disease severity using stepwise regression, principal component regression and partial least-square regression. Journal of Zhejiang University SCIENCE B, 8(10), 738-744.

Zhu, L., Zhang, H., Zeng, F., Zheng, K., Zhang, X., Hu, H., \& Jin, Q. (2011). Estimation of rice neck blasts severity using spectral reflectance based on BP-neural network. Acta Physiologiae Plantarum, 33(6), 2461-2466. 